\title{
La construcción de la masculinidad en el Colegio Militar Leoncio Prado (1944-2014)
}

\author{
José Smith Vásquez Mendoza \\ jsvam8123@gmail.com \\ Universidad Peruana de Ciencias Aplicadas \\ Universidad de Lima
}

\section{RESUMEN:}

El tradicional Colegio Militar Leoncio Prado ha formado durante los últimos setenta años a un gran número de hombres bajo un sistema de internado y una férrea disciplina castrense. Este tipo de educación que parece exagerada y extrema no está muy alejada de la formación que recibimos los hombres en nuestros hogares, vecindarios y colegios civiles.

Por tal razón el presente estudio pretende exponer la forma en que se construye la masculinidad en la sociedad limeña-en el tiempo-a través de una institución de encierro disciplinaria. Para saber cómo han variado y que constantes se han mantenido con los años, y comprender que la violencia en la sociedad está directamente relacionada con la educación que recibimos los hombres desde muy jóvenes.

Palabras Clave: Género; Masculinidad; Educación; Militares.

\section{Building Masculinity in the Leoncio Prado Military High School (1944-2014)}

\begin{abstract}
:
The traditional Military High School of Leoncio Prado has trained a large number of male students formed the last seventy years based on a strong educational militaristic system. This type of education that seems exaggerated and extreme shows some similiraties to the education that young males receive either at home, their neighbohoods, or publich schools. This study tries to present the way in which the masculinity is constructed in the society of Lima offered by boarding school educational system. Also, it is explored what relationship exists between violence in society and education that young males have received in their social contexts as a whole.
\end{abstract}

Keywords: Gender; Masculinity; Education; Military.

(C) Los autores. Este artículo es publicado por ISHRA, Revista del Instituto Seminario de Historia Rural Andina de la Facultad de Ciencias Sociales de la Universidad Nacional Mayor de San Marcos. Este es un artículo de acceso abierto, distribuido bajo los términos de la licencia Creative Commons Atribucion - No Comercia _ Compartir Igual 4.0 Internacional. (http://creativecommons.org/licenses/by-nc-sa/4.0/) que permite el uso no comercial, distribución y reproducción en cualquier medio, siempre que la obra original sea debidamente citada. 


\section{Vásquez \\ ¡Leonciopradinos...! \\ Alto el pensamiento como una bandera, \\ encendida el alma como azul hoguera, recio el corazón.}

Hagámonos dignos del nombre que lleva nuestra Institución.

(Coro del himno del CMLP)

\section{Introducción}

Los estudios de género se han desarrollado en gran abundancia, y nacen como tal, por el impulso que las feministas le dieron a este tipo de investigaciones durante el siglo XX. Entonces no es casualidad que la mayoría de los trabajos estén dirigidos a conocer el mundo de las mujeres y sus complicaciones sociales frente a los hombres.

Esas investigaciones unidas a una fuerte lucha política y social lograron que sean oídas, consigan un mayor espacio en la sociedad, y obtengan -en algunos casos-las mismas oportunidades de los hombres, entre otras conquistas. Sin embargo, los principales causantes del sometimiento, discriminación, e injusticias hacia las mujeres no hemos recibido un análisis similar porque se cree que al estudiar lo femenino ya se está haciendo referencia a su opuesto lo masculino (Fuller, 1997).

Ello trajo como resultado un menor número de estudios sobre los hombres y la formación de su masculinidad; así como un limitado análisis de la forma cómo lograr sociedades más equitativas tanto para hombres como para mujeres.

Por lo tanto, es necesario analizar los espacios e instituciones donde los hombres aprendemos a desarrollarnos como tales. De esta manera comprenderemos porque la masculinidad está ligada a una serie de prohibiciones en lo referente a nuestras emociones y sentimientos, pero a su vez con la necesidad de exponer constantemente competencia, violencia, valentía, superioridad, fuerza y liderazgo (Callirgos, 1998).

Así como una forma de aportar a los estudios de género, en el presente trabajo estudiaremos al tradicional Colegio Militar Leoncio Prado, (en adelante CMLP) quien ha formado durante los últimos setenta años a un gran número de hombres bajo un sistema de internado y una férrea disciplina castrense. Este tipo de educación que parece exagerada y extrema no está muy alejada de la formación que recibimos los hombres en nuestros hogares, vecindarios, clubes deportivos, universidades, colegios civiles, entre otros espacios sociales. Por tal razón, nos hemos planteado un conjunto de preguntas que intentaremos responder en las siguientes páginas:

¿Cómo se construye la masculinidad en los adolescentes del CMLP? ¿Por qué los valores militares son apreciados por la sociedad peruana? ¿Por qué existe la necesidad de educar a los hombres bajo un sistema disciplinario donde deben mostrar constantemente su hombría? ¿Hay convergencia entre valores masculinos de la sociedad civil y de la institución militar? Y ¿Qué consecuencias puede traer para la sociedad una educación exclusiva para hombres y con una dirección militar?

Nosotros consideramos como Kimmel (1992), Callirgos (1998) y otros autores que la masculinidad es un proceso histórico que se va construyendo en la sociedad a partir de un conjunto de permanencias y tradiciones conservadas en diversas instituciones. Por ello en el presente trabajo ratificaremos esta postura tomando de modelo al CMLP, ya que es un espacio de 
formación de adolescentes donde se pretende inculcarles valores tales como el amor a la patria, fuerza, coraje, valentía, honor, disciplina entre otros.

El periodo de estudio serán los últimos setenta años de vida institucional que van de 1944 a 2014. Tiempo que ayudará a comprender cómo la masculinidad es un proceso histórico que se construye en base a varias generaciones. El trabajo está estructurado en dos partes; en la primera se describirá a la institución en estudio, el Colegio Militar Leoncio Prado (CMLP) fundación, funcionamiento y tradición. En la segunda se expondrá cómo se construye la masculinidad en el CMLP, a través del proceso de adaptación de los cadetes en tercero, cuarto y quinto año. Todo ello a partir de fuentes escritas, gráficas y orales. Estas últimas son entrevistas realizadas a cadetes y ex cadetes de diferentes promociones del Colegio, será a partir de sus testimonios y experiencias que conoceremos como se construye la masculinidad en este espacio de encierro.

\section{El Colegio Militar Leoncio Prado}

El contexto internacional de la Segunda Guerra Mundial (1939-1945) y sobre todo el triunfo peruano en el conflicto con el Ecuador (1941) trae consigo no solo réditos políticos para el presidente de la época Manuel Prado, sino, principalmente una reivindicación a la milicia peruana venida menos por las derrotas del pasado. Respecto al tema, Contreras y Cueto (2013) reflexionan lo siguiente:

La victoria militar se convirtió en un triunfo político para las Fuerzas Armadas, que tenían sobre sí el fantasma de la derrota de la guerra con Chile del siglo XIX, y para el propio presidente Prado, a cuya familia se le criticaba abiertamente porque su padre, el presidente Mariano Ignacio Prado, abandonó el país a poco de iniciada la Guerra del Pacífico (p. 293).

Ese fue el escenario propicio para desarrollar el proyecto de un colegio militar para el país. Ya que existían serias deficiencias en los postulantes a las instituciones militares peruanas (Sierralta, 1969).

Uno de los primeros en mencionar la necesidad de la creación de una institución educativa para menores de dirección militar fue el entonces comandante EP Juan Mendoza Rodríguez, quien desde hacía unos años antes y en conferencias en el Círculo Militar, expuso la necesidad de mejorar el nivel académico de los postulantes a las instituciones militares a partir de hacer incidencia en sus estudios previos (Villacorta, 1956, p.43) al haberse notado serias deficiencias académicas y, por ende, registrase un alto número de desaprobados por parte de los postulantes a las Fuerzas Armadas (Sierralta, 1969, p.17).

Elaborado el proyecto y con apoyo directo de los comandantes de las FF.AA. se logra redactar y aprobar el Decreto Ley del 27 de agosto de 1943, promulgado por el presidente Manuel Prado Ugarteche y el ministro de educación, Sr. Elías La Rosa. Aquél dispositivo legal dio a luz al primer Colegio Militar Nacional para formar a jóvenes estudiantes en una elevada cultura cívica y militar básica (Sierralta, 1969, p. 31). Esta institución, bajo un sistema de régimen especial de preparación, fue denominada Leoncio Prado por la Ley N. 9890 y promulgada por el Congreso de la República el 18 de enero de 1944.

La institución recibió ese nombre no solo porque el héroe de la batalla de Huamachuco (1882) es medio hermano del presidente de aquellos años, sino primordialmente porque es un personaje que su vida refleja independencia y un alto interés por la vida militar. Desde los tempranos ocho años combinó sus estudios escolares con su participación en la escuela militar, luego se involucra 
en diferentes sucesos históricos como el combate del Dos de mayo de 1866 con tan solo 13 años, participa en el motín del Colegio Guadalupe a los 14, la lucha por la independencia de Cuba a los 20, realiza una travesía por las Filipinas a los 24, y toma parte activa en el conflicto con Chile de 1979 a los 26 años. Leoncio Prado es un personaje cuyo coraje, valores patrióticos y amor por la libertad, son ensalzados como el ejemplo de hombre fuerte, viril y nacionalista que el Estado pretendía construir a partir de un nuevo estilo de educación militarizada.

Se designó como primer director del colegio al coronel EP José Del Carmen Marín Arista, quien realizará la primera convocatoria para el concurso público de admisión de la nueva institución a inicios del año 1944. En Lima se realizaron las inscripciones en las instalaciones del colegio nacional Alfonso Ugarte y en provincias se realizó el mismo acto en los principales colegios de cada ciudad (Gómez, 1993, p. 48). La propuesta era seleccionar a los mejores estudiantes del país para que este modelo educativo se desarrollase con gran éxito. Al parecer la idea de una educación de dirección militar fue muy bien acogida por los padres de familia ya que hubo un gran número de postulantes (Sierralta, 1969, p.18). Lamentablemente e igual que en otras ocasiones, a pesar de las intenciones por ejecutar el proyecto y las grandes expectativas de la población, el propio Estado mina al proyecto al no haber designado un presupuesto para su implementación, tampoco se tenía un local para su funcionamiento. Por tal razón, el coronel Marín en su calidad de Director del plantel, decide tomar la iniciativa en la búsqueda de un espacio donde se inicien las labores escolares, encontrando así un viejo cuartel -a medio construir- conocido con el nombre de La Chalaca, el cual estaba ubicado en los acantilados del Callao, un espacio abandonado y convertido en basural, sin la infraestructura adecuada para iniciar de forma adecuada las labores de formación educativa (Gómez, 1993, pp. 48-49). A pesar de las dificultades descritas, la dirección decidió tomar el lugar para instalar ahí al Colegio Militar al no haber otro inmueble disponible. A estos altibajos, se debe describir las tardías gestiones de las autoridades pertinentes para el envío de recursos económicos y materiales, no sorprendiendo a pocos que los primeros cadetes del colegio, quienes se habían internado el 22 de mayo de 1944, tuvieran que sufrir de las carencias de infraestructura y logística, ayudando al mismo tiempo en la limpieza y adecuación de aquel edificio a medio construir. Por esa razón la primera promoción egresada del colegio recuerda con mucho aprecio el inicio de las actividades académicas al afrontar estos retos insospechados para un estudiante y aún con ello, salir adelante frente a los problemas y adversidades, hecho que algunos ex cadetes marcan como el «espíritu de los leonciopradinos» (Sierralta, 1969, pp. 18-20).

Superadas las dificultades, el 15 de julio de 1944 se desarrolla la fundación oficial del Colegio Militar Leoncio Prado, acontecimiento que concentró a la principales autoridades del país y a un importante conjunto de delegaciones como la de los cadetes de la Escuela Militar de Chorrillos, la Escuela Naval de La Punta, la Escuela Aeronáutica Jorge Chávez y la Escuela de la Guardia Civil y Policía; además de estudiantes de los grandes colegios públicos de Lima tales como los de Nuestra Señora de Guadalupe, Alfonso Ugarte, José Granda, Pedro Labarthe y el Dos de Mayo (Sierralta, 1969, p. 34). La reunión de estas instituciones militares y civiles mostraba la importancia y aceptación de la innovadora forma de educación que se estaba creando en el Perú, en la que, además de impartir conocimientos en un alto nivel, se seguiría una vida estricta, disciplinada y bajo una formación militar que pretendía producir estudiantes con ideales patrióticos, generando no solo militares mucho mejor preparados, sino también nuevos ciudadanos para el fortalecimiento de la nación, al modo que pretendía la Ley Orgánica de Educación Pública del año 1941, tal cual se argumentó en los considerandos para la creación del CMLP en 1943: 
Que de acuerdo con lo establecido en el artículo $94^{\circ}$ de la Ley Orgánica de Educación Pública, la educación en el país tiene como objetivos principales la cultura, la salud, el desarrollo físico, la moralidad y el nacionalismo de los educandos (...) es necesario intensificar por todos los medios posibles el espíritu civico de la juventud en el país, cultivándolo en ideales patrióticos y en las virtudes ciudadanas, y preparándolo para su noble ejercicio (Prospecto del CMLP 1944, p. 55)

La ley mencionada nos revela como nuevamente todo lo relacionado a la milicia vuelve a tomar importancia en la sociedad peruana, situación que ya durante el siglo XIX se había presentado con el ascenso de los caudillos y las diversas guerras internacionales que construyen -en el país- un ideal de hombre fuerte, viril, sacrificado y patriota. Un modelo aceptado y apreciado por un buen sector de la población.

La formación educativa en el CMLP comprendía -y se mantiene hasta nuestros días- tres años de estudios, iniciando en tercero y culminando en el quinto de secundaria, tiempo en el que recibirían una educación de calidad y se nutrirían de la disciplina castrense. Terminado el proceso, los primeros cien puestos del colegio tenían la posibilidad de ingresar directamente a las Fuerzas Armadas, inclusive a las principales universidades y escuelas civiles (Sierralta, 1969, pp.19-20). El beneficio académico generó gran prestigio a la institución y un mayor número de postulantes -no solo de Lima o el Callao, sino de diferentes lugares del país- acudían por ganarse el derecho a ingresar a sus aulas. Estos estudiantes surgían desde condiciones sociales diferentes $\mathrm{y}$ de formaciones culturales diversas que, en aquellos pabellones que miraban a los acantilados del Callao, se reunían en un espacio de encierro para lograr un reconocimiento y, en muchos casos, su ascenso social.

El CMLP fue un pertinente laboratorio de prueba para poder experimentar con diversas propuestas pedagógicas que años después serán aplicadas por a nivel nacional por el Ministerio de Educación. Un ejemplo de lo descrito fue el proyecto de las grandes Unidades Escolares edificadas durante el gobierno del presidente Manuel A. Odría (1950 -1956), en cuyo gobierno tuvo como ministro de educación pública al coronel EP Juan Mendoza Rodríguez, uno de los creadores y ex director del CMLP. Por ello estas nuevas instituciones educativas civiles tuvieron varias características del colegio militar enumerando algunas como el uso de un uniforme muy similar al del ejército, la disciplina férrea, diversas actividades patrióticas y deportivas, infraestructura muy bien diseñada, y la separación de los estudiantes por géneros en diversos planteles.

Entre los años 1977 y 1980, correspondientes a la segunda fase del Gobierno Revolucionario de las Fuerzas Armadas, el CMLP pasa por una de sus crisis más importantes cuando el gobierno de turno decide transformarlo de su estatus de nivel Secundario especial a una Escuela Superior de Educación Profesional (ESEP) en el contexto de la Reforma Educativa de 1972. Tal cambio tuvo como principal consecuencia el alejamiento de profesores de nivel universitario, reemplazados por docentes de colegios públicos. El proyecto pretendía generar estudiantes que egresen con una profesión técnica y luego, si los mismos alumnos lo decidían, podían continuar con una carrera de nivel superior. Sin embargo, la propuesta no fue bien ejecutada ni tampoco bien recibida por la población. La consecuencia inmediata fue el masivo número de retiros y deserciones que llegaron a 150 cadetes para el año 1978 (Collantes, 1993, pp. 55-70). En la década de los ochentas del siglo pasado, el colegio intenta recuperarse académicamente pero factores externos como las crisis económicas experimentadas en los gobiernos de Fernando Belaunde (Partido Acción Popular) y principalmente el de Alan García (Partido Aprista Peruano) no permitieron mayores avances; a lo que se debe sumar la violencia terrorista que generó diversos problemas financieros 
y logísticos al Estado, teniendo como consecuencia el dejar a un lado a la educación nacional al ser priorizada la lucha por la seguridad política y económica interna. Así se llega a la última década del siglo XX durante el gobierno del Ing. Alberto Fujimori, quien durante su segundo mandato (1995-2000) extiende sus políticas neoliberales a la educación, promulgando una serie de decretos como los del año 1996 que permitían el ingreso de inversionistas privados al sector educativo. Esta decisión logra masificar el acceso a los colegios privados con una oferta académica de dudoso nivel (Cuenca, 2013, pp. 38-39). Además, ese mismo año el CMLP se adaptó al nuevo Proyecto Educativo Nacional (NEP) que disponía una mayor participación civil en la institución, flexibilizándose las normas para su ingreso, mayores derechos para los cadetes e intervención de los padres de familia. Estos cambios no fueron acompañados de un mejoramiento académico ni de un mayor apoyo económico o de infraestructura para la institución. Una vez más el número de postulantes e ingresantes se redujo, marcando una nueva crisis institucional. El promedio de ingresantes al CMLP fue de 500 a 600 cadetes, pero durante la aplicación del proyecto ESEP (1978-1980) se reducen en promedio entre 400 a 280. Luego en los ochentas las cifras suben entre a 500 a 450, pero durante los noventas vuelven a reducirse el número de ingresantes a cifras entre los 400 y 250 cadetes. (Libros de matrícula del CMLP)

Por último, ya iniciando el nuevo siglo y durante el segundo gobierno de Alan García se crea el programa de Colegios Emblemáticos del Perú (2009) para mejorar la infraestructura de los grandes y más antiguos centros educativos del país, proyecto donde se incluyen a las Grandes Unidades Escolares y al CMLP. Por tal razón y dos años después, se realiza el traslado momentáneo del colegio al Fuerte Hoyos Rubios donde desarrollará sus funciones hasta finales de 2012 (Lam, 2017). Aquí los escolares del CMLP vivirán la experiencia de estudiar en un fuerte militar y convivir con la tropa y los oficiales durante dos años. Hacia el año 2013 ya estaban listas las nuevas instalaciones del CMLP y entonces vuelven a su local tradicional, pero con nuevas instalaciones y con los ánimos de recuperar la trayectoria perdida. Sin embargo, ahora deberán competir con otro tipo de institución de encierro fundada en el año 2010. El Colegio Mayor Secundario Presidente del Perú, institución civil que reúne a los mejores estudiantes del país y que, bajo un sistema de internado, pretende ser un ejemplo pedagógico para una posterior aplicación nacional. Aquí se busca otra mirada en la construcción del ciudadano y la ciudadana, una más libre, menos rígida que la militar, pero con altos conocimientos y desarrollos pedagógicos para producir estudiantes preparados para una vida académica superior.

El CMLP desde 1944 hasta el 2014 ha generado 68 promociones con un aproximado de 50,700 cadetes educados bajo un fuerte rigor militar (Libros de matrícula CMLP). Esta breve historia institucional que aquí hemos presentado, nos describe cómo en un momento de la historia peruana el ideal de hombre civilizado estuvo relacionado con la milicia y como ello fue variando lentamente en el tiempo. Aunque todavía en nuestros días persiste la idea, en muchas familias peruanas, de una educación para sus hijos con influencia militarizada y de fuertes valores patrióticos; y es por eso que una institución tan tradicional como el CMLP continúa existiendo, razón por la cual en otros lugares del país se han seguido construyendo colegios militares durante el siglo XXI. Asíen zonas andinas y de la selva se fundaron las instituciones; Gregorio Albarracín (Tacna), Pachacútec Inca Yupanqui (Cusco), Inca Manco Cápac (Puno), Basilio Auqui (Ayacucho), Domingo Nieto (Ilo), Héroes del Cenepa (Bagua), Ancco Hayllo (Andahuaylas), Rafael Hoyos Rubios (Cajamarca) y Juan Valer Sandoval (Huancayo).

\section{III.- La construcción de la masculinidad en el CMLP}

Iniciaremos este estudio a partir de las reflexiones del investigador Michel Kimmel (1992) quien en su libro La producción Teórica sobre la masculinidad, dice que la masculinidad «no viene 
en nuestro código genético, tampoco flota en una corriente del inconsciente colectivo esperando ser actualizada por un hombre en particular, o simultáneamente por todos los hombres». La masculinidad argumenta: «se construye socialmente: 1. desde una cultura a otra, 2 . en una misma cultura a través del tiempo, 3. durante el curso de la vida de cualquier hombre, 4 . entre diferentes grupos de hombres según clase, raza, grupo étnico y preferencia sexual» (p. 135).

Entonces las formas de ser hombre son una construcción socio-cultural desarrollada a través del tiempo y en diversas instituciones. En este trabajo ratificaremos esta postura dando como ejemplo al CMLP, el cual es una institución donde muchas familias peruanas confiaron la formación de sus hijos, porque se promueve la disciplina, moralidad y trabajo. Además de darles la capacidad de independencia, valentía y coraje; necesaria no solo para la vida militar sino en la sociedad.

La construcción de la masculinidad en el CMLP se desarrolla en el transcurrir de los tres años que duran los estudios en esta institución. Cada año representa una etapa de superación que va acompañada de retos y dificultades, de ahí que cada parte de este proceso tiene un animal como símbolo del periodo por el cual se ha logrado superar.

Por esa razón se explicará esos periodos de la siguiente manera: se inician siendo «perros» en tercer año; luego superado este proceso duro, pasarán a ser «chivos» en cuarto año; y finalmente serán «vacas» en quinto año.

\section{a) Tercer año, Los perros}

Los nuevos cadetes, de trece y catorce años de edad en promedio, tienen un internamiento de tres meses alejados de sus familias con derecho a visita los domingos por unas horas. A partir de este momento serán llamados perros, porque deberán serán fieles, obedientes, respetuosos y seguir las órdenes de los oficiales y los monitores. Estos últimos son alumnos de 5to año capacitados para ser los guías de los iniciados.

Los ingresantes pasan por una ceremonia de bienvenida donde se presentan con sus ropas civiles, y se alejan de la protección de sus familias. Este momento se parece mucho al vivido por los niños espartanos cuando son arrancados de los brazos de sus madres para ingresar a la milicia. Así, entre llantos y despedidas las madres ven a sus hijos alejarse para convertirse en hombres.

Juan Carlos Callirgos (1998) expone que «la mayoría de sociedades considera necesario desligar a los varones adolescentes de sus madres, pues seguir unido a ellas implicaría ser femenino» (p.44). Entonces,la importancia de esta ceremonia radica en simbolizar la ruptura con el mundo femenino representado por la madre y el inicio de su aprendizaje para una vida independiente siendo hombre.

Terminado el protocolo serán llevados a conocer sus cuadras o dormitorios; recibirán sus uniformes, se les cortará el cabello, todo bajo violencia verbal y castigos físicos si no se obedecen a tiempo y bien las órdenes.

Este es el inicio del proceso donde la mayoría de los nuevos cadetes comprenden que deben adaptarse rápidamente a la nueva forma de vida regida por la disciplina, obediencia, rigor, horarios y pocas horas de sueño. Características de lo que Erving Goffman (2001) 1lama «instituciones totales» porque pretenden controlar toda la vida de los individuos que están sometidos a su régimen totalizador. 
Los primeros días son importantes pues aquí dentro del grupo de lo ingresantes se formarán los líderes, sus seguidores y los que no logran adaptarse. Estos últimos ingresaron al colegio obligados por sus familiares; y si no consiguen ser respetados serán los que más sufran humillaciones de sus compañeros y superiores. Para mostrar lo expuesto narraré lo contado por el ex cadete llamado Raúl (Promoción XLII-1988):

La mañana siguiente de internarnos nos despertaron a las cinco de la mañana, rápidamente a tender las camas, y a las duchas, ahi todos estábamos desnudos excepto Alex, quien estaba bajo la ducha en ropa interior con una mirada de miedo. Todos lo observaban y empezaron a burlarse de él. De pronto llegó el monitor y preguntó por qué el alboroto. Entonces le contamos que había un perro bañándoseen calzoncillo... el monitor se acercó a él y ordenó que entre todos le rompiéramos la ropa interior...y así se hizo. Alex terminó llorando, desnudo y humillado. El monitor al verlo le dijo: Aquí todos somos hombres, no debe existir vergüenza ... ya no estás en tu casa... (Raúl, comunicación personal, 14 de abril de 2011)

Como muestra el breve relato desde los primeros días se les enseña, a través de la humillación, lo que un hombre no debe sentir, ni hacer. El pudor entre varones no está permitido, porque todos son iguales y nadie debe ocultar su cuerpo, pues hacerlo refleja debilidad e incluso homosexualidad. Además una característica de la masculinidad es el poder sobre otros, quien exprese debilidad será inferiorizado y sometido por los demás hombres hasta que demuestre estar al mismo nivel.

Similares situaciones se evidencian en el mismo espacio de las duchas donde compararán sus penes; quienes tengan el mayor tamaño serán respetados y los de menor extensión, degradados y constantemente se les recordará su «debilidad».

Al respecto Callirgos señala que la condición masculina está siempre en duda, por lo que necesita someterse a pruebas y a una afirmación constante para ser aceptado socialmente (1998, p. 30). No realizar lo que hacen todos los hombres, siendo varón, te deja en una postura de desconfianza porque se cree se está ocultando algo.

En el primer año en el CMLP, los perros pasaran por diversas jornadas de maltratos y castigos con números ejercicios físicos. Sin embargo, podemos mostrar que en el transcurso de este periodo hay tres ritos o sucesos que marcarán la estadía de los nuevos cadetes en su camino a convertirse en hombres.A continuación explicaremos brevemente cada una de ellas:

El primero es el llamado bautizo, ceremonia que algunas veces fue pública y presenciada por los padres y familiares, entendida como una bienvenida al colegio y parte del proceso para que sus hijos se desarrollen como hombres. Consistía en un desfile donde los perros eran disfrazados de diversos personajes; y donde los cadetes de cuarto y quinto año se burlaban de ellos obligándoles a realizar cualquier tipo de acciones ridículas, así como pintarlos. Todo bajo la aceptación de las autoridades militares y los familiares.Sin embargo, el verdadero recibimiento sucedía la última noche de internamiento antes de la salida de los perros, luego de los tres meses de encierro. Tal suceso ya no contaba con la presencia de familiares pero tenía el aval de las autoridades militares.

Cada excadete tiene su propia versión de lo que ahí sucede, en general se puede señalar un alto grado de violencia ejercida por los cadetes de quinto año, quienes toman las cuadras por la noche para someter a los perros a diversos maltratos en los dormitorios, baños e incluso en 
el estadio del colegio. Para explicar lo ocurrido tomaremos el testimonio de Luis (Promoción XXXV-1978):

...Todos ya habíamos sido advertidos de lo que sucedería esa noche, por lo tanto nos alistamos para recibir a las vacas (cadetes 5toaño) doble ropa por dentro y bloqueamos las puertas de los dormitorios con los camarotes para impedir el ingreso... eran las cero, cero horas cuando empezó todo, llegaron ellos como mucha fuerza y violencia tratando de ingresar con múltiples amenazas y maldiciones nos exigían que abriéramos las puertas... ante nuestra negativa decidieron romper las puertas, una vez que lo lograron se inició el bautizo. Cada cadete de quinto año tomó a uno de nosotros y empezó a golpearlo y a obligarnos a realizar diversos actos como lamer las losetas, golpear a otro compañero, beber agua de los inodoros... una vez que se cansaron de hacer eso nos dijeron que nos desnudemos y que nos vayamos al estadio. Ahi nos pidieron que nos metamos a unos hoyos recientemente escavados. Luego de eso nos arrojaron tierra y nos empezaron a orinar y a escupir, burlándose de nosotros, diciendo bienvenidos al infierno, donde solo los verdaderos hombres sobreviven...(Luis, comunicación personal, 27 de abril de 2011)

Lo contado por Luis no se aleja mucho de la versión dada en 1963 por Mario Vargas Llosa en su libro La Ciudad y los perros:

...lo desnudaron y la voz le ordenó nadar de espaldas, sobre la pista de atletismo...Después lo volvieron a una cuadra de cuarto tendió muchas camas y cantó y bailó sobre un ropero, imitó a artistas de cine, lustró varios pares de zapatos, barrió la loseta con la lengua, fornicó con una almohada, bebió orines... la cuadra estaba silenciosa, los muchachos se miraban unos a otros y, a pesar de haber sido golpeados, escupidos, pintarrajeados y orinados, se mostraban graves y ceremoniosos...(2001, p. 48)

Este suceso violento puede ser comparado con los ritos tribales de iniciación de los adolescentes hombres en diversas culturas en el mundo, ya que los hombres al no tener un inicio biológico exacto de su madurez sexual -como en las mujeres la menstruación- necesitan marcar a través de una ceremonia el inicio de su hombría exponiendo su valor y resistencia (Callirgos, 1998, p. 44)

El antropólogo David Gilmore ha estudiado diversos ritos de iniciación hombres jóvenes en el mundo. A partir de ello considera que las culturas buscan en los varones demostraciones y representaciones de «hombres de verdad» apoyados en una doctrina viril del logro y bajo presión social $(1994$, p. 215).

La mayoría de cadetes y ex cadetes entrevistados en esta investigación consideran al bautizo un momento especial porque lograron vencer el dolor y la humillación. Algunos como Julio (Promoción XLVI-1990) piensan que ese momento difícil le enseñó a enfrentar retos más complejos en su vida, o el momento donde toda la promoción se unió según César (Promoción XLIX-1993). Podemos sintetizar que esta primera experiencia les dio a los cadetes de tercer año la sensación de haberse convertido en hombres viriles, fuertes, valientes, dejando atrás al niño protegido por la familia.

Los excesos en este rito consiguieron varias veces la prohibición de tremendos actos. Uno de los primeros intentos por suprimirlos se dio en 1950, unos días después al bautizo de Mario Vargas Llosa, donde el cadete Valderrama terminó con las yemas de la mano izquierda cortadas. Al siguiente año las autoridades del colegio decidieron realizar una gincana a modo de «bautizo oficial» (Vilela, 2011, pp.37-38). Al parecer estas actividades no calmaron los bautizos violentos 
porque en 1958 el director, coronel Wilfredo Blondet, los prohibió una vez más (Sierralta, 1968, p. 58). De igual forma se ordenó en 1979 y 1998, la eliminación de cualquier acto de violencia contra los cadetes ingresantes. Sin embargo, el tradicional bautizo no se ha dejado de practicar en los setenta años de la institución, solo tornó a ser una actividad menos pública, semi-escondida de las autoridades, con menor violencia y las cuadras como lugar exclusivo para su realización.

El siguiente acontecimiento importante es la ceremonia cívico-religiosa de la entrega de armas; aquí el cadete luego de haber superado dificultades y aprendido la instrucción básica militar recibirá de las manos de su padre o madre un fusil Máuser.

El acto protocolar es muy similar al que se realiza en las escuelas militares, se desarrolla en el patio central del colegio; los cadetes visten sus uniformes de campaña y los padres van vestidos formalmente. La ceremonia se inicia con una liturgia encargada al capellán militar, quien bendice las armas; a continuación se llamará a un familiar (generalmente son las madres) para entregar el fusil a su hijo. Luego al unísono las madres dirán «consérvenlas con honor», y de esa forma concluye esta ceremonia muy emotiva y simbólica a la vez, ya que los padres entregan a sus hijos a la patria. Aquí es muy importante observar la consolidación de tradiciones donde se permite la continuidad del ejercicio del poder de los hombres, ligados a las guerras, violencia, honor y valentía. De esta forma se van concretizando la masculinidad en los adolescentes del CMLP.

Para finalizar este primer año los perros deben demostrar su resistencia en una caminata de campaña en el desierto, aquí se pondrán a prueba sus capacidades de sobrevivencia y obediencia a las normas. Una vez más se deberá exhibir la masculinidad. Quienes logren pasar el reto serán respetados y quienes se quejen o no resistan las dificultades de la jornada terminarán maltratados y humillados.

Gilmore (1994) explica que la masculinidad se trata de «una virilidad condicionada por situaciones adversas y precarias para sobrellevar la escasez de recursos y que es fomentada para contrarrestar el impulso universal de huir ante el peligro» (p.229). Es decir, frente a circunstancias críticas, los verdaderos hombres, en colaboración colectiva, superarán los momentos de desgracia, pues son los únicos capaces de conseguir lo imposible.

Las jornadas de caminatas de los cadetes en el desierto son una experiencia única donde se les somete a situaciones extremas como tener poca agua y comida, deberán demostrar que son hombres. Además, la ocasión es propicia para - por fin - usar los fusiles que se les asignaron a través de una simulación de combate donde se les permitirá disparar a blancos marcados en lomas y cerros. La experiencia para mayoría de cadetes es inolvidable; algunos de ellos cuentan lo poderosos que se sentían al tener un arma de aniquilamiento en sus manos (Mario, Promoción XXXI-1974). Una vez más, surgen entre sus recuerdos los padecimientos y sufrimientos en esas jornadas, pero dicen valió la pena porque les demostró hasta dónde podía llegar y lo capaces que pueden ser.

Para terminar se va a transcribir un lema para subir la moral de los cadetes de tercero, contado por el excadete César (Promoción LXIII, 2009) quién recibió estas palabras de aliento de su monitor de quinto año luego de la marcha de campaña:

Hoy son perros, mañana aspirantes, y pasado mañana monitores o cadetes de quinto año...

El otro año vienen otros perros, ustedes tendrán que dar el ejemplo, ya estarán solos...

$Y$ el siguiente año estarán en quinto, los mejores serán monitores, y tendrán sus perros y comandarán su compañía...Perros, ;todo pasa y todo llega! (César, comunicación personal, 8 de mayo de 2013). 
Entonces la tradición de la violencia se transmite de generación en generación sin que nadie note algo malo porque son hombres los que se están formando y es esta forma lo quelos caracteriza como tales.

Este primer año el número las bajas o retiros del colegio por diversas circunstancias pueden llegar a cincuenta, es decir el $20 \%$ de los cadetes que ingresaron al Leoncio Prado (Libros de matrícula CMLP). Ello puede señalar un proceso donde un número importante de hombres no están de acuerdo con el trato y rudeza con el que fueron sometidos, este rechazo no tiene por qué convertirlos en varones de segunda categoría pues naturalmente los hombres no nacemos con algún tipo de gen de violencia y resistencia. Sin embargo, los perros que pidieron su salida del colegio serán vistos como inferiores, hijos de mamá que no lograrán desarrollarse con éxito en la vida.

Los que lograron superar este primer nivel se sentirán orgullosos de sí mismos y le darán mayor valor a su género al considerar que están hechos para una vida de valentía y liderazgo. Además esperan con grandes ansias el 4to año porque ya no estarán al mando de los alumnos de 5 to y por lo tanto serán más independientes.

\section{b). Cuarto año, Los chivos}

En cuarto año los cadetes son llamados chivos, no hay un consenso de la razón del nombre. Sin embargo, algunos leonciopradinos relacionan al animal caprino con los cambios físicos de los adolescentes de 14 y 15 años. Esos cambios son los primeros bigotes-barbas y voces agudas similares a las de ese animal. Otros piensan que al estar en cuarto año y tener el derecho de defenderse y quejarse ante los abusos de los cadetes de último año se trata de ironizar su capacidad de tener voz para enfrentarlos; comparándolos con los chivos y su balar.

Es importante señalar que en cuarto año el colegio permite el ingreso de alumnos de otros colegios militares o civiles. Esos estudiantes serán llamados cuervos, por ser considerados como aves intrusas o pueden ser traidores al no haber pasado el bautizo con los demás miembros de la promoción. Ello implica no tener la mística de los ritos y ceremonias pasadas en tercer año, es decir ellos no vivieron «la perrada», no son parte de la manada masculina. Por lo tanto tendrán que hacer muchos méritos si quieren ser aceptados como parte de la promoción.

Para entender la lógica de los cadetes de cuarto año se expondrá el testimonio de Marcos, un leonciopradino de la promoción 2013 quien luego de haber superado su primer año en el colegio reflexiona y nos cuenta:

cuando terminó el año me di cuenta que todo pasa y todo llega; cuando aguantamos un año de masacre... Y ahora ya estoy aquí, ahora veremos asomándonos en nuestras ventanas a los perros que ingresen al Colegio Militar Leoncio Prado, como salen a formar a las cuatro de la mañana para poder desayunar y nosotros salir a formar tranquilos y ver a los perros con sueño al iguallo que pasamos nosotros...Bueno pasamos muchas cosas; cosas que se pueden contar y otras que no... Ahora nos toca gozar que al otro año vienen nuestros perros y también los masacraremos...» (Marcos, comunicación personal, 10 de mayo de 2013)

Aquí se observa el empoderamiento y orgullo que sienten los cadetes de este nivel por haber superado momentos difíciles. Además se notan las ansias de disfrutar de la libertad y el mando a futuro, ya no serán ellos los maltratados, sino otros, eso los llena de satisfacción, se sienten viriles.

En este año se inician una serie de competencias para demostrar que son hombres completos. Esas competencias pasan por las comparaciones físicas musculares, deportivas, militares y la pérdida de la castidad sexual por cualquier medio. 
En cuanto a las competencias castrenses quienes tengan mayores actitudes de coraje, valor marcialidad para desarrollar los aprendizajes militares serán admirados por sus compañeros, más aún si son elegidos para participar junto a los cadetes de quinto año en la delegación que el colegio presente en la Parada Militar de 29 de julio. Formar parte del batallón de representación del colegio es uno de los momentos de mayor huella en los estudiantes, pues se llenan de emoción y de orgullo, algunos incluso le brillan los ojos cuando cuentan su experiencia en ese desfile (Pedro, Promoción LXIII, 1976).

En lo referente a la iniciación sexual muchos de ellos recurrieron de forma grupal a burdeles en el Callao, La Victoria y el Centro de Lima. Lo sucedido en aquellos lugares serán las principales historias al retornar al colegio, luego de los dos días de descanso. Otros expresarán que no necesitan ir a los lupanares porque lo hacen con la enamorada de turno o alguna amiga a la cual le hicieron promesas sentimentales para lograr su objetivo.

Entonces se ingresa a una nueva etapa de las relaciones con el género opuesto, marcándose el valor que las mujeres tienen según la facilidad a la que accedan para mantener relaciones sexuales. Así una chica que rápidamente acceda al sexo será vista como inferior y no se le tomará en serio. Por lo contrario si se hace «respetar» y no permite el acto sexual será considerada superior comparada con otras mujeres. En el colegio algunos relacionan a las mujeres y sus virtudes de acuerdo a la clase social. Al respecto Norma Fuller en su libro Identidades masculinas, investigó sobre las relaciones de los géneros en clases medias y altas, de la sociedad peruana, llegando, entre otras conclusiones, a explicar que el registro de la imaginación masculina revela los diferentes estilos de relación intergéneros que los varones pueden emprender de acuerdo a la clase social de las mujeres con las que se relacionan. Entonces una mujer de sector popular es vista como fácil, libidinosa o una persona poco respetable; pero si la condición económica y social mejora los hombres de las clases acomodadas las tratan con mayor respeto, pero sin dejar de lado el hecho de verse como superiores frente a ellas (1993, p. 43). Hecho muy similar al pensamiento de algunos leonciopradinos frente a su relación con las mujeres, quienes prefieren para su iniciación sexual buscar a una chica humilde o de barrio porque están seguros que con ellas será mucho más sencillo y fácil lograr su objetivo de perder la castidad.

Otros sucesos durante el cuarto año están relacionados con el consumo de bebidas alcohólicas, las primeras borracheras e incluso uso de drogas. Dichas acciones contribuyen a generar una imagen de virilidad, fuerza y resistencia frente a otros cadetes que se negaron a realizar dichas acciones. Si bien en el CMLP se promueve una educación donde la disciplina y los valores son muy importantes, es necesario explicar que en paralelo y sin la presencia de una autoridad, los cadetes se sienten lo suficientemente libres como para tomar sus propias decisiones y asumir sus propios riesgos para demostrar qué tan valientes, audaces y avezados pueden ser para romper las normas o burlarse de la autoridad. Ello forma parte de esa necesidad subconsciente de demostrar constantemente qué tan hombre son a sus compañeros y la sociedad. A continuación relataremos tres experiencias:

Ernesto (Promoción LXIII, 1997) Nos contó cómo burlaban la vigilancia del colegio para escaparse alguna fiesta o prostíbulo lo que le llaman «tirar la contra», principalmente ocurría los miércoles o jueves y esperaban la media noche para salir de su cuadra y luego trepar las paredes para conseguir por unas horas de libertad. Él nos cuenta que se sentían unos héroes y sus egos se inflaban al poder cometer con éxito tamaña hazaña; comenta con orgullo que lo hicieron más de diez veces sin ser sorprendidos. 
Por su parte José (Promoción LXVIII, 1992). Describe que durante cuarto año tuvieron varias borracheras, las cuales eran muy bien organizadas; desde el domingo de ingreso al internamiento llevaban ron y pisco en botellas de plástico como si fueran gaseosas, luego elegían una madrugada donde sabían que el encargado de vigilar la cuadra era sobornable o también iba a participar del consumo, además otro criterio para elegir cuándo lo harían era conocer el curso y profesor con el que tendrían clases horas después, ello era muy importante porque de preferencia elegían una donde el docente era poco estricto o lo podían burlar para poder dormir durante sus horas de clase. Una vez coordinado todo ello se reunían en los baños o en algún lugar dentro de la cuadra a beber mientras se contaban historias sobre alguna pena o aventura amor. Según José, fueron momentos de alegría y de sentirse orgullosos - como grupo - al haber logrado ir en contra de lo establecido sin ser descubiertos.

Para finalizar tenemos el testimonio de Enrique (Promoción LXIII, 2007-2009) quien dice que dentro del colegio hubo varios consumidores de drogas, pero nadie los acusaba porque era el grupo de chicos «rudos», además peor que consumir drogas era acusar a un compañero, «los soplones» terminaban mal, eran considerados traidores y poco hombres por todos los cadetes.

Como se observa a pesar de tener una educación disciplinada y dirigida desde una perspectiva militar, los estudiantes desarrollaron una doble moral; una para cuando están al frente de las autoridades que respetaban, y otra para los espacios privados o carentes de alguien que los vigile. Una actitud reprobable pero que aprendieron no solo de sus compañeros de años superiores, sino de algunos militares a su mando. Entonces construyen su comportamiento en la sociedad actuando de dos formas; una pública donde siguen las reglas y parecen seres humanos correctos, y otra donde pueden burlar a la autoridad y actuar con mayor libertad.

Esa doble moral que resaltamos es parte de las características de la masculinidad tradicional de un hombre peruano e incluso nos atreveríamos a decir latinoamericano, debido a que se difunde con gran amplitud la idea de que un hombre no revela secretos, ni anda contando lo vivido dentro de una agrupación, porque sería un soplón o chismoso, cualidad otorgada a las mujeres vistas como inferiores. Además es importante señalar que esta característica no solo se aplica a lo militar, sino a los deportes, principalmente el fútbol, los grupos de amigos, clubes, e incluso claustros religiosos.

En la misma línea la trasgresión de la norma y la autoridad, es parte de ser masculino, porque un hombre debe constantemente demostrar que es hombre sin importar los riesgos y los peligros a los cuales pueda someterse (Callirgos, 1998, pp. 27- 30). Así el CMLP refuerza esas ideas machistas, tanto en su educación formal, como en lo aprendido en el ámbito privado, donde hay una constante competencia por exponer al otro su mayor nivel de masculinidad.

Los dos años de compañerismo en el colegio generan fuertes lazos de amistad entre los miembros de la promoción quienes se estiman y quieren, pues han pasado por diversas circunstancias críticas. Sin embargo, las demostraciones de cariño no son libres como en el caso de las mujeres, quienes no sienten vergüenza de abrazar y besar a sus similares para expresar el aprecio de su amistad. Para el caso de los leonciopradinos y hombres peruanos en general el aprecio por otro hombre se demuestra a través de la violencia, la ofensa entre otras acciones rudas.

Al respecto Tarnawiecki Barrio, sostiene a partir de su estudio sobre los hombres desde su estado emocional y psíquico, que los varones debemos negar nuestros sentimientos y emociones para ser considerados como tales. Es decir, se nos ha prohibido llorar, expresar afecto, ternura 
(1993, p. 58). Este proceso no es fácil porque debemos tener mayor control de nuestro cuerpo, de lo que sentimos y no que debemos expresar. Por lo tanto se usan los mecanismos de miedo, burla o broma para camuflar lo que se nos está prohibido.

Este año también termina con la marcha de campaña por los arenales de Lima y el Callao, pero los cadetes de cuarto año caminan en otras condiciones pues ellos ya pasaron por todas las pruebas y sacrificios. Ahora les toca burlarse de los perros de tercero, quienes sufren y luchan por demostrar que son lo suficientemente hombres para seguir en el colegio, aunque todavía no tienen el prestigio y respeto que poseen los cadetes de quinto año, por eso esperan con muchas ansias el siguiente año para poder comandar el colegio.

\section{c). Quinto año, Las vacas}

La última etapa de este proceso hacia la construcción de la masculinidad en el CMLP se desarrolla en el quinto año de secundaria. Aquí los cadetes son llamados vacas, según varios leonciopradinos se debe al significadode sacralidad que les dan en la India a estos mamíferos, otros piensan que se deben a que comen y viven bien, además de estar robustos y sanos. Entonces ser alumno de quinto año debe representar un ser sagrado, intocable, con gran respeto y prestigio.

Dentro de los cadetes de quinto se elegirá a una élite llamados monitores, quienes serán seleccionados por sus actitudes para la vida militar, buenas calificaciones y su deseo de sacrificar su verano de vacaciones para poder participar de cursos de capacitación. Este grupo tendrá jornadas duras de entrenamiento básico militar, como paracaidismo, comando, sobrevivencia entre otros. Pero el principal objetivo de esta preparación es enseñarle al cadete-monitor a dirigir y gobernar el colegio con cierta autonomía.

Terminadas las capacitaciones los monitores y cadetes de quinto año se internarán una semana antes de que se inicien las clases. Su sueño desde tercer año está por cumplirse, serán literalmente los que gobiernen el colegio, en cuanto el orden y la disciplina. Muchos cadetes consideran el mejor momento dentro del colegio, como Erick (Promoción XX, 1963). Quien esperó con muchas «ganas» este año porque deseaba mandar a otros, y hacer pasar a los perros lo mismo que él vivió.

Percy (Promoción XXXVI, 1979) considera llegar a quinto como un momento de gloria, no solo porque mandaba en el colegio, sino que sentía haber madurado, dejando atrás al niño mimado.

Fernando (Promoción XXXIX, 1983). Por el contrario considera que el llegar a quinto año fue un alivio, ya que terminaba esa etapa de su vida tan dolorosa, nos comenta su decisión de no ejercer violencia contra ningún estudiante de tercero porque sentía no era necesario para dar conocer lo aprendido en el CMLP.

Rubén (Promoción LXVI, 1990). Tiene sentimientos encontrados cuando piensa en su llegada a quinto año, pues dice se sentía orgulloso de haber superado los dos primeros años, pero a la vez considera que tal vez menos dureza hacia ellos le hubiera hecho pasar una mejor adolescencia.

Por último, Alex (Promoción LXVII, 2011), comprende el proceso de esos años como necesarios en su formación. «Ingresé como un chico tímido y con poca confianza, y ahora soy todo lo contrario, hasta he cambiado físicamente y tengo muchas "habilidades sociales"》». 
El término del quinto año es un momento muy triste para la mayoría de los cadetes pues representa el fin de una etapa de su vida donde aprendieron a desarrollarse y a ser independientes junto con jóvenes de su misma edad y alejados de la protección familiar, es decir hacerse hombres bajo los criterios de la sociedad peruana.

Solo el 15 o $20 \%$ de los egresados del CMLP continúan sus estudios superiores en alguna de las escuelas de las Fuerzas Armadas peruanas. La gran mayoría busca nuevos rumbos para su futuro en el mundo civil, espacio para al que están preparados ya que el proceso para ser hombre no es muy distinto en otras instituciones, la diferencia se encuentra en que los leonciopradinos logran desarrollar completamente su masculinidad en tres años, lo que a otros varones les puede tomar mayor tiempo.

\section{Conclusiones}

Como se ha observado la masculinidad no es una condición biológica sino una construcción social histórica que está basada en un conjunto de tradiciones transmitidas de generación en generación. Para el caso del CMLP se ha construido en el transcurso de sus más de setenta años de vida institucional, y durante tres etapas; donde el tercer año es un estado fundamental para definir quiénes son «verdaderos hombres», porque cada día, semana y mes, el cadete deberá superar diversas pruebas encaminadas a la construcción de su masculinidad. Además, una vez superada esa etapa, de obediencia y retos, durante el cuarto y quinto año se va formando en los estudiantes un carácter de mando y dirección, pero también la búsqueda de independencia asumiendo riesgos que incluso van en contra de las reglas de la institución.

El proceso de masculinidad en el CMLP, aunque puede parecer duro, cruel y violento, no es tan distinto a lo desarrollado en otras instituciones educativas y sociales, pues en el camino para ser hombre la sociedad exige la separación de lo femenino y se supriman las emociones que lo puedan hacer ver como débiles. La principal diferencia entre el colegio militar y otros centros educativos es que aquí el proceso de aprendizaje para ser hombre es más rápido, apenas tres años, situación que en otras realidades puede demorar un mayor tiempo.

El gran número de familias que ha confiado la educación de sus hijos al CMLP nos muestra que la sociedad peruana ha construido una forma de ser hombre ligada a las duras condiciones de la vida militar, tal vez ahí radique el éxito y duración - en el tiempo - de una institución de más de setenta años. Donde, miles de jóvenes han formado su comportamiento bajo valores de disciplina, valentía y virilidad, pero a su vez han desarrollado un carácter para enfrentar a una sociedad que exige un comportamiento distinto tanto para el ámbito público como el privado.

El CMLP refuerza las ideas machistas construidas en el mundo civil; un hombre debe saber autocontrolarse, pero a su vez debe constantemente demostrar que lo es a través de actos de valentía, rudeza, incluso trasgredir a la autoridad y la norma sin importarle las consecuencias. Además, en ningún caso deberá acusar a sus compañeros o colegas, porque si no será señalado como traidor, soplón, chismoso, y ello lo vincularía con el mundo femenino interiorizándolo y perdiendo su estereotipo masculino. Así, el mundo de los hombres construye una doble moral, una para la vida pública; disciplinada y ordenada, y otra para el ámbito privado; donde libera sus represiones y hace uso de su libertad para sentirse macho.

Una educación militarizada como la impartida en el Leoncio Prado puede generar hombres con mayor amor a la patria y de gran cultura cívica, sin embargo mantiene las tradicionales diferencias patriarcales entre hombres y mujeres, que producen una sociedad injusta, violenta, y donde los seres humanos no somos realmente libres porque debemos seguir asumiendo los 
roles establecidos, históricamente, por una sociedad empecinada en mantener comportamientos artificiales.

Aprender sobre masculinidad nos sirve para comprender por qué existe tanta violencia en nuestra sociedad. Y cómo prevenir-corregir en el futuro la crianza de los hijos e hijas. Es pues necesario un cambio en las tradiciones y costumbres donde se nos permita a los hombres desarrollarnos sin suprimir nuestras emociones y donde lo femenino no sea visto como inferior sino en las mismas condiciones que lo masculino. Necesitamos un mundo donde los varones podamos demostrar amor, aprecio y valor sin que ello signifique represión personal o el sacrificio de otras personas para conseguirlo.

\section{Bibliografía}

Barrio, T. (1993). Reflexiones sobre la masculinidad. Pretestos (5),Lima: DESCO.

Barrig, M. (1981). Convivir, la pareja en la pobreza. Lima: Mosca Azul editores.

Callirgos, J. (1996). Sobre Héroes y Batallas Los caminos de la Identidad Masculina. Lima:Escuela para el desarrollo Demus.

Cárdenas, L. (1997). La Construcción de la Masculinidad en Sectores Populares: El caso de jóvenes cargadores de La Parada. Lima: EuroPROFEM.

Díaz, P. (1999). El Colegio Guadalupe y la educación peruana. Lima: Edit. Mantaro.

Fuller, N. (1997). Identidades masculinas.Lima: PUCP

Gilmore, D. (1994) Hacerse hombre, concepciones culturales de la masculinidad. Barcelona: Ediciones Paidós.

Goffman, E. (2008). Internados: Ensayos sobre la situación social de los enfermos mentales. Buenos Aires: Amorrortu editores.

Gómez, Oscar (1993) El primer eslabón de oro. Lima:Editorial Prisma.

Kimmel, M. (1992). La producción teórica sobre la masculinidad. Nuevos aportes. Isis internacionales; México: Ediciones de las mujeres $\mathrm{N}^{0} 17$.

Lam, J. (2017) CMLP: de Colegio Emblemático a Colegio de Alto Rendimiento. Gaceta Leonciopradina (272), Recuperado en 4 de julio de 2017 de http://www.gacetaleonciopradina.com/?p=5027.

León, R.y Stahr M. (1995). Yo actuaba como varón solamente. Entrevistas a procesados por delito de violación. Lima: DEMUS.

Melhus, M. (1993). Una vergüenza para el honor, una vergüenza para el sufrimiento.

Quito: Ediciones Abya-Yala.

Romero, T. (1993) Colegio Militar Leoncio Prado; Bodas de oro. Lima: CMLP.

Sierralta, A. (1968). Historia del Colegio Militar Leoncio Prado. La Perla: CMLP.

Tauro, A. (2001) Enciclopedia Ilustrada del Perú. Tomo 11. Lima: Editorial Peisa.

Vilela, S. (2011). El cadete Vargas Llosa. Jaén: Alcalá Grupo editorial.

Villacorta, A. (1956) Breve historia del Colegio Militar Leoncio Prado. La Perla: Editorial CMLP.

Presentado: 16 septiembre 2017

Aceptado: 5 febrero 2018

Publicado online: 27 febrero 2018 